\title{
Succession secondaire et perte de diversité végétale après réduction du broutage dans un pâturage boisé des Alpes centrales suisses
}

\author{
François Freléchoux ${ }^{1,4}$, Marco Meisser ${ }^{2}$ et François Gillet ${ }^{3}$ \\ ${ }^{1}$ Institut fédéral de recherches WSL, Station 2, CH-1015 Lausanne; \\ e-mail: francois.frelechoux@wsl.ch \\ 2 Station de recherches Agroscope Changins-Wädenswil ACW, CP 1012, CH-1260 Nyon 1 \\ ${ }^{3}$ Laboratoire des systèmes écologiques, Ecole Polytechnique Fédérale de Lausanne, Station 2 , \\ CH-1015 Lausanne \\ ${ }^{4}$ Laboratoire d'Ecologie et Evolution, Département de Biologie, Pérolles, Chemin du Musée \\ 10, $\mathrm{CH}-1700$ Fribourg
}

Manuscrit accepté le 28 mars 2007

\begin{abstract}
Freléchoux F., Meisser M. and Gillet F. 2007. Secondary succession and loss in plant diversity following a grazing decrease in a wooded pasture of the central Swiss Alps. Bot. Helv. 117: $37-56$.

Reduced cattle grazing pressure in the Alps has caused the reforestation of many subalpine pastures during the last decades. To understand the dynamics of natural reforestation and to evaluate how this change affects plant species diversity, we described the vegetation of a wooded pasture in the central Swiss Alps (Sembrancher, Valais) using the integrated synusial method. Based on stratified vegetation relevés in 27 plots, we defined 11 community types at the synusial level (two tree-layer, five shrublayer, and four herb-layer synusiae), and four community types at the phytocoenosis level (pasture, tall forbs and scrub, wooded pasture and forest). The spatial distribution of these four phytocoenoses suggests that they represent successional stages after abandonment, and that the pathway of vegetation succession depends on the aspect. We suppose that on northern oriented, cool and shady locations, abandoned pastures first develop towards tall-forb meadows and scrub with Alnus viridis, and then to a preforested stage with Picea abies and Larix decidua. In contrast, on western oriented, warm and sunny location, Larix decidua (mainly) and Picea abies directly colonize the abandoned pastures, but further succession finally leads to the same pre-forested stage as on northern slopes. Plant species richness was highest in open areas and decreased by $25 \%$ as tree cover increased from $6 \%$ to $65 \%$. According to our successional model, plant species diversity is lost more rapidly on northern slopes (with species-poor green
\end{abstract}


alder scrub) than on western slopes (with species-rich young larch forests), suggesting that northern slopes most urgently need an appropriate grazing management.

Key words: Abandonment, stocking rate, succession, synusial phytosociology, plant diversity, sustainability.

\section{Introduction}

Dans toute l'Europe, la forêt s'installe rapidement en zone de montagne. En Suisse, la surface forestière a augmenté de 47’000 ha (ou 4\% ) entre 1985 et 1995 (OFS/OFEFP 2004). Près de 23'000 ha d'alpages ont disparu entre les périodes 1979/85 et 1992/97 au profit de zones d'arbustes et de broussailles (38.4\%), ou de forêt (35.1\%) (OFS 2005). Plus de la moitié de l'accroissement de la surface forestière est intervenue entre 1400 et 2000 m d'altitude et l'accroissement relatif a été maximal entre 2200 et 2400 m, c'est-àdire vers la limite supérieure de la forêt. Cette dynamique forestière importante peut être mise en rapport avec la diminution du cheptel bovin. En Valais par exemple, les effectifs sont passés de 63'000 têtes en 1936 à 33'000 têtes en 1993, soit une diminution de près de moitié (Fellay 1998).

Les transformations des paysages alpins sont liées aux changements des activités agricoles (Tasser et Tappeiner 2002). Le nombre d'exploitations a fortement diminué et si les terres agricoles proches des villages ont subi une certaine intensification (p. ex. par l'apport d'engrais minéraux ou l'arrosage), les parcelles les plus éloignées ont souvent été utilisées de façon plus extensive, voire simplement abandonnées. Des cultures en terrasses et des prés de fauche ont été délaissés. L'activité pastorale s'est aussi fortement réduite de la zone des mayens à celle des pâturages subalpins et alpins, entraînant une très forte dynamique d'invasion des ligneux.

Ces transformations rapides du paysage ont des conséquences sur la diversité végétale et animale. Avec la disparition des terrasses, la flore adventice qui leur était liée s'est fortement raréfiée (Werner 1988). L'abandon des prés de fauche non fertilisés ou leur reconversion en pâturage mettent en péril la grande biodiversité qu'ils hébergent (Baur et al. 2006; Fischer et Wipf 2002). La diminution du cheptel d'estivage et l'abandon des pâturages les plus éloignés entraîne un fort embroussaillement et réduit la richesse biologique qu'elles abritent. Il est urgent de suivre cette dynamique et d'en évaluer les conséquences écologiques.

En Suisse, les principales espèces arborescentes de l'étage subalpin sont l'épicéa (Picea abies, ci-dessous Picea), le mélèze (Larix decidua, ci-dessous Larix), l'arolle (Pinus cembra), les pins de montagne (pin à crochet, Pinus uncinata et pin rampant, Pinus mugo). Picea est très fréquent et occupe surtout les stades terminaux de la dynamique forestière. Larix est une espèce pionnière qui affectionne les sols minéraux et de bonnes conditions de lumière en régions peu embrumées jusqu'à la limite supérieure de la forêt (Ozenda 1985). Larix est souvent favorisé par l'homme au détriment de Picea, car il fournit un bois d'œuvre de qualité tout en favorisant le pâturage, grâce à son interception modérée de la lumière (Delarze et al. 1998). Il contribue ainsi à maintenir la flore des milieux ouverts et à augmenter la production fourragère dans les régions sèches (Werner 1988). Pinus cembra est une espèce pionnière, tolérante au stress thermique (gel en particulier) et qui occupe la partie supérieure de l'étage subalpin. Pinus cembra et Larix dominent une association forestière classique de l'étage subalpin supérieur des Alpes internes: le Larici - Pinetum cembrae (Ozenda 1985; 
Favarger 1995; Oberdorfer 1992). Les pins de montagne sont des espèces pionnières et fréquentent les terrains calcaires ou siliceux; ils diffèrent par leur distribution, occidentale pour Pinus uncinata, orientale pour P. mugo (=P. mughus Scopoli). Dans le Parc national des Grisons, l'abandon de la pâture a permis l'installation de Pinus uncinata précédant l'installation du Larici - Pinetum cembrae (Risch et al. 2004). La lande à Rhododendron ferrugineum occupe la frange supérieure de l'étage subalpin; elle constitue le sous-bois du Larici-Pinetum cembrae, mais elle s'élève aussi au-delà de la limite supérieure de la forêt. La distribution de cette lande peut être considérée d'une part comme naturelle car cette espèce résiste mieux que les arbres aux rudes conditions climatiques et d'autre part comme artificielle car elle résulte des déboisements et de l'action du bétail qui ont maintenu ces zones ouvertes (Favarger 1995). L'aulne vert (Alnus viridis) est une espèce buissonnante qui affectionne les endroits frais et humides, sur sol bien aéré et riche en terre fine (Favarger 1995). Son optimum se situe dans le subalpin inférieur et son développement est maximal dans les Préalpes du Nord (Ozenda 1985). Par l'action du bétail, il a été relégué dans les couloirs à avalanches et les pentes très humides (Richard 1990; Anthelme et al. 2001), mais il constitue aussi un stade évolutif menant à la forêt de conifères (Favarger 1995).

Un grand nombre d'interrogations persistent quant à la dynamique de la végétation et aux relations bétail-végétation à l'étage subalpin et à sa frange supérieure. Par endroits, les landes subalpines, p. ex. celles à Rhododendron ferrugineum ou à Juniperus communis var. nana, semblent revêtir un caractère envahissant suite à une diminution de la pression de broutage. De même, Alnus viridis montre un très fort potentiel d'installation dans les pâtures abandonnées car il se reproduit de façon sexuée comme végétative (Anthelme et al. 2002). Ses peuplements denses, très couvrants, avec une biomasse et une productivité primaire élevées (Wiedmer et Senn-Irlet 2006), peuvent fortement réduire la biodiversité (Anthelme et al. 2001). Dans les Alpes internes, notamment en Valais, Larix et Pinus cembra jouent un rôle prépondérant dans la succession secondaire à l'étage subalpin. Il y a lieu d'évaluer l'emprise de cette dynamique, de la décrire, de la localiser précisément dans le paysage. Il est nécessaire d'évaluer la perte de diversité biologique comme celle des structures de la végétation et des éléments d'un paysage façonné par l'homme depuis des siècles. Dans une perspective de conservation de ces milieux et des espèces qu'ils hébergent, il est nécessaire de bien comprendre l'action du bétail spécifique aux différentes espèces ligneuses envahissantes en relation avec la pression de broutage (Vandenberghe et al. 2007).

Notre présente contribution a pour but de (i) décrire la végétation d'un site expérimental et proposer des schémas dynamiques conduisant des milieux ouverts à la forêt en intégrant notamment l'effet du bétail, (ii) évaluer les diversités spécifique et structurelle de la végétation au cours de son évolution.

\section{Matériel et Méthodes}

\section{Site d'étude}

L'alpage étudié se trouve sur la commune de Sembrancher (Valais, Suisse) au lieudit le Larzay (46 03' 41' N, 7 ${ }^{\circ} 10^{\prime}$ '53' E; Fig. 1a). L'altitude varie entre 1420 et 2020 m. Le site subit l'influence d'un climat à tendance continentale (Werner 1988) avec peu de brouillard. A la station météorologique la plus proche (Mauvoisin, à $14 \mathrm{~km}$ du site, avec une altitude de $1841 \mathrm{~m}$ ), la somme annuelle des précipitations pour la période 1972 à 
1995 s'élève à $1187( \pm 159)$ mm en moyenne $( \pm 1 S D)$ dont $867( \pm 203)$ mm sous forme de neige. La température de janvier s'élève pour la même période en moyenne à $-3.9( \pm$ 2.3 ${ }^{\circ} \mathrm{C}$ et celle de juillet à $11.1( \pm 1.7)^{\circ} \mathrm{C}$. Le site comprend une partie exposée à l'ouest, relativement sèche, et une partie exposée au nord, nettement plus fraîche. Le substrat géologique est formé de matériaux mixtes (calcaires bleutés et conglomératiques, schistes noirs et quartzites, flysch calcaire) à dominance siliceuse (Burri et al. 1983).

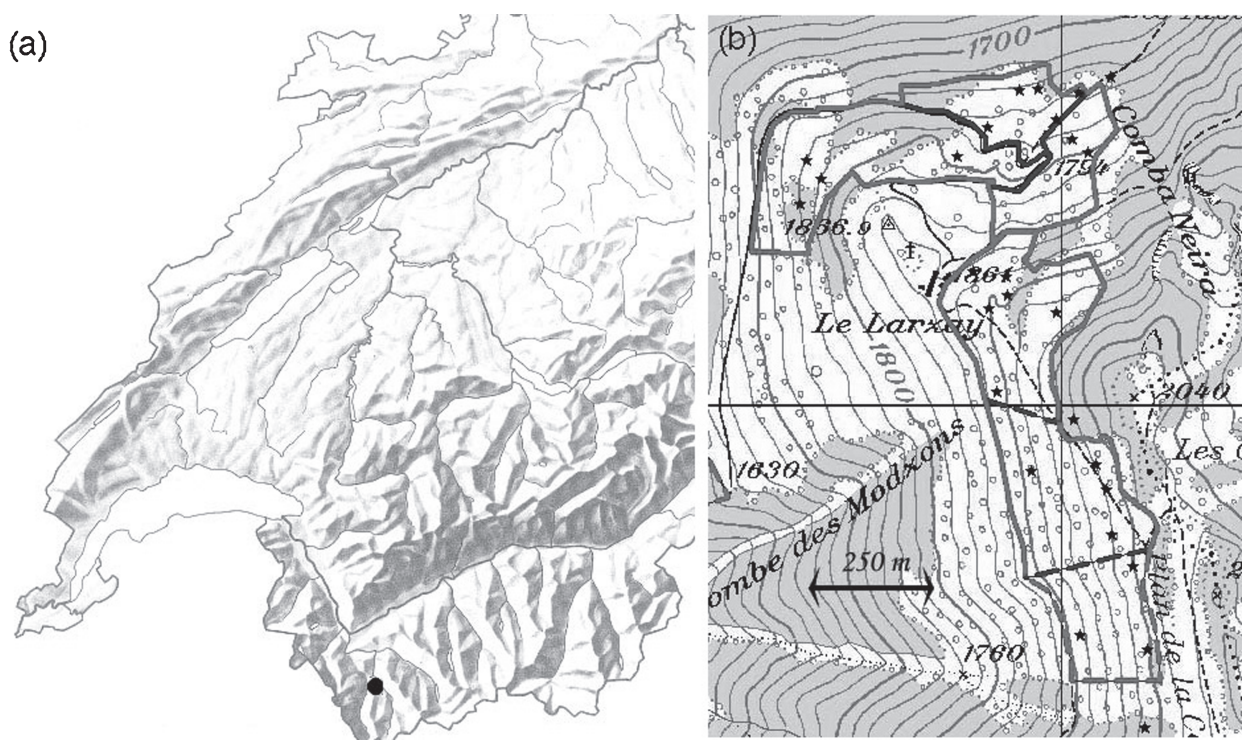

Fig. 1. Pâturage du Larzay: (a) situation en Suisse et (b) périmètre d'étude avec les six parcs utilisés par le bétail dans la zone supérieure du pâturage. L'emplacement des 27 relevés phytocénotiques est noté par des étoiles. Carte (b) reproduite avec l'autorisation de swisstopo (BA068288).

La pression de broutage a fortement diminué depuis quelques décennies, ce qui a initié un développement important des recrûs de Larix et d'Alnus viridis. Dès 2005, les surfaces marginales de l'alpage, sur les pentes les plus fortes et dans les secteurs les plus reculés et les plus embroussaillés, ont été utilisées pour le projet PASTO (www.srva.ch/ pages/development_rural.htm; http://ecos.epfl.ch/indexrecherche.en.php), alors que les terrains les plus favorables au bétail continuaient d'être occupés par un autre troupeau. Depuis 2005, 18 vaches et 9 veaux de la race d'Hérens sont estivés sur le site de début juin à fin septembre. La zone d'observation s'étend sur 26 ha et comprend six parcs qui se trouvent dans la partie supérieure de l'alpage (Fig. 1b).

\section{Échantillonnage de la végétation}

La phytosociologie synusiale intégrée (Gillet et al. 1991; Gillet et Gallandat 1996) est une approche qui vise à décrire la structure hiérarchique des communautés végétales et les relations spatiales et temporelles entre ses différents composants. Parmi les différents niveaux d'organisation spatio-temporels, nous avons considéré les deux premiers niveaux: (1) la synusie qui est une communauté végétale concrète unistrate liée à des conditions environnementales homogènes; (2) la phytocénose qui est un 
complexe de synusies étroitement liées structurellement et fonctionnellement. A partir d'une description de la structure spatiale (stratification, mosaïque) des phytocénoses, la méthode permet de générer des hypothèses sur les modalités et le déterminisme de la dynamique de la végétation.

En été 2005, 27 placettes (Fig. 1b), dont la surface est comprise entre 400 et $900 \mathrm{~m}^{2}$, ont été sélectionnées préférentiellement (exposition nord et ouest, gradient de boisement) pour décrire les unités de végétation représentées sur le site, selon des critères d'homogénéité structurelle et écologique. Dans chaque placette, la phytocénose a été décrite par une liste de synusies végétales, dont chacune a fait l'objet d'un relevé floristique séparé, selon un échantillonnage préférentiel utilisant les critères classiques d'homogénéité floristique et d'aire minimale empirique. 115 relevés synusiaux ont ainsi été effectués, soit 23 relevés de synusies arborescentes (ligneux de plus de $8 \mathrm{~m}$ de hauteur), 53 relevés de synusies arbustives (ligneux dont la hauteur est comprise entre $50 \mathrm{~cm}$ et $8 \mathrm{~m}$ ) et 39 relevés de synusies herbacées. Pour les synusies arbustives, un seuil de hauteur à $1.30 \mathrm{~m}$ a été considéré pour séparer les synusies arbustives basses ("b"), formées de sous-arbrisseaux et du recrû soumis à l'abroutissement, et les synusies arbustives hautes ("B") qui constituent le stade fourré du peuplement ligneux hors d'atteinte du bétail.

L'appréciation du recouvrement relatif (ou contribution, avec la somme égale à 1) des espèces dans chaque synusie et du recouvrement (surface relative) des synusies dans chaque phytocénose a été réalisée par estimation visuelle de la dominance selon les codes de Braun-Blanquet (1964). La nomenclature des plantes vasculaires se réfère à la première édition de Flora Europaea (Tutin et al. 1964-1980).

\section{Traitement des données}

La typologie des synusies arborescentes a été obtenue par un tri manuel du tableau de relevés. La typologie des synusies arbustives et herbacées a été réalisée au moyen de groupements agglomératifs hiérarchiques avec R 2.3.1 (R Development Core Team 2006) et la librairie 'cluster'. Avant le traitement des relevés des synusies herbacées, le recouvrement relatif des espèces dans chaque relevé a été transformé par la racine carrée pour diminuer le poids des espèces dominantes. Les groupements (Legendre et Legendre 1998) ont été effectués en appliquant la méthode de Ward (minimum variance clustering) à une matrice de distances de corde (chord distance). Le nombre de groupes a été choisi en considérant le profil des silhouettes (Rousseeuw 1987). Les groupes ainsi obtenus ont été assimilés à des syntaxons élémentaires ou types de synusies (Gillet et al. 1991). La fidélité des espèces à chaque syntaxon a été ensuite calculée par l'indice IndVal (Dufrêne et Legendre 1997) qui tient compte de la dominance (recouvrement relatif).

La typologie des phytocénoses a été réalisée après avoir défini l'ensemble des types de synusies. Les analyses ont été effectuées sur les recouvrements absolus des syntaxons élémentaires dans les 27 relevés phytocénotiques avec les mêmes options que pour la typologie des synusies. Les groupes de relevés obtenus ont été assimilés à des coenotaxons élémentaires ou types de phytocénoses. Dans chaque coenotaxon, la proportion moyenne des groupes socio-écologiques d'espèces herbacées (espèces caractéristiques des syntaxons synusiaux de prairies de fauche, de prés pâturés gras, de pelouses maigres, d'ourlets maigres, de friches, de sous-bois et de marais ou prairies humides) a été calculée à partir des relevés synusiaux en se basant sur le référenciel syntaxonomique de Julve $(1993,2006)$. 
Pour comparer la diversité végétale des syntaxons, la richesse floristique (nombre d'espèces de plantes vasculaires par relevé synusial) et la régularité des relevés ont été calculées. La régularité (evenness), calculée comme le rapport entre la diversité de Simpson du relevé et le nombre d'espèces (Gillet et al. 1999), représente la composante de la diversité liée aux différences de dominance; elle varie entre ca. 0 (disparités extrêmes dans la dominance des espèces) et 1 (aucune différence de dominance). A l'échelle des phytocénoses, les comparaisons ont porté sur la diversité synusiale (diversité de Shannon calculée sur la liste des syntaxons synusiaux par phytocénose, Gillet and Gallandat 1996) et sur le nombre total d'espèces de plantes vasculaires, toutes strates confondues.

Enfin, les relations spatiales (contacts, superpositions) et floristiques (stades de développement des ligneux, similarités entre relevés) observées entre les syntaxons herbacés, arbustifs et arborescents ont servi à élaborer deux diagrammes de l'organisation spatio-temporelle des principaux types de phytocénoses, permettant de reconstituer les liens dynamiques supposés qui existent entre les synusies et entre les phytocénoses, en soulignant principalement ceux qui sont influencés par les activités du bétail.

\section{Résultats}

La typologie des synusies a abouti à la définition de 11 types de synusies ou syntaxons élémentaires, caractérisés par leur composition floristique, leur physionomie, leur écologie et leur répartition dans le terrain d'étude. De la même façon, quatre types de phytocénoses ou coenotaxons élémentaires ont pu être définis.

\section{Typologie des synusies arborescentes}

La strate arborescente est le plus souvent dominée, sur les versants ouest et nord, par des peuplements plus ou moins denses de grands (15-25 m de haut) Larix (Tab. 1, syntaxon A1). Les peuplements sont très clairsemés à proximité de l'écurie et sont plus denses à plus grande distance, notamment en direction du sud. Les souches observées au sol dans la zone proche de l'écurie témoignent de coupes réalisées dans le passé.

Le second groupement arborescent (Tab. 1, syntaxon A2), qui montre une constance de Larix et de Picea, représente, en exposition ouest et nord, un peuplement de transition entre le mélézein et la pessière subalpine.

\section{Typologie des synusies arbustives}

Les synusies arbustives jouent un rôle important dans la dynamique du boisement qu'elles soient soumises à l'action du bétail («b») ou non («B»). Larix et Picea forment un important recrû dans la zone d'étude, parfois accompagné, en exposition nord, par Alnus viridis (Tab. 1, syntaxon b4+B4). En situation nord, rarement ouest, on trouve un groupement dominé par Alnus viridis avec une bonne fréquence de Picea, mais dans lequel Larix est peu représenté (Tab. 1, syntaxon b5+B5). Les synusies arbustives dominées par Picea, parfois accompagné de Larix, se trouvent en situation pré-forestière ou forestière et en orientation nord et ouest (Tab. 1, syntaxon b3+B3).

A côté des synusies basses du recrû (b3, b4, b5), on trouve les synusies arbustives de landes dominées par Rhododendron ferrugineum ou par Juniperus communis ssp. nana, 


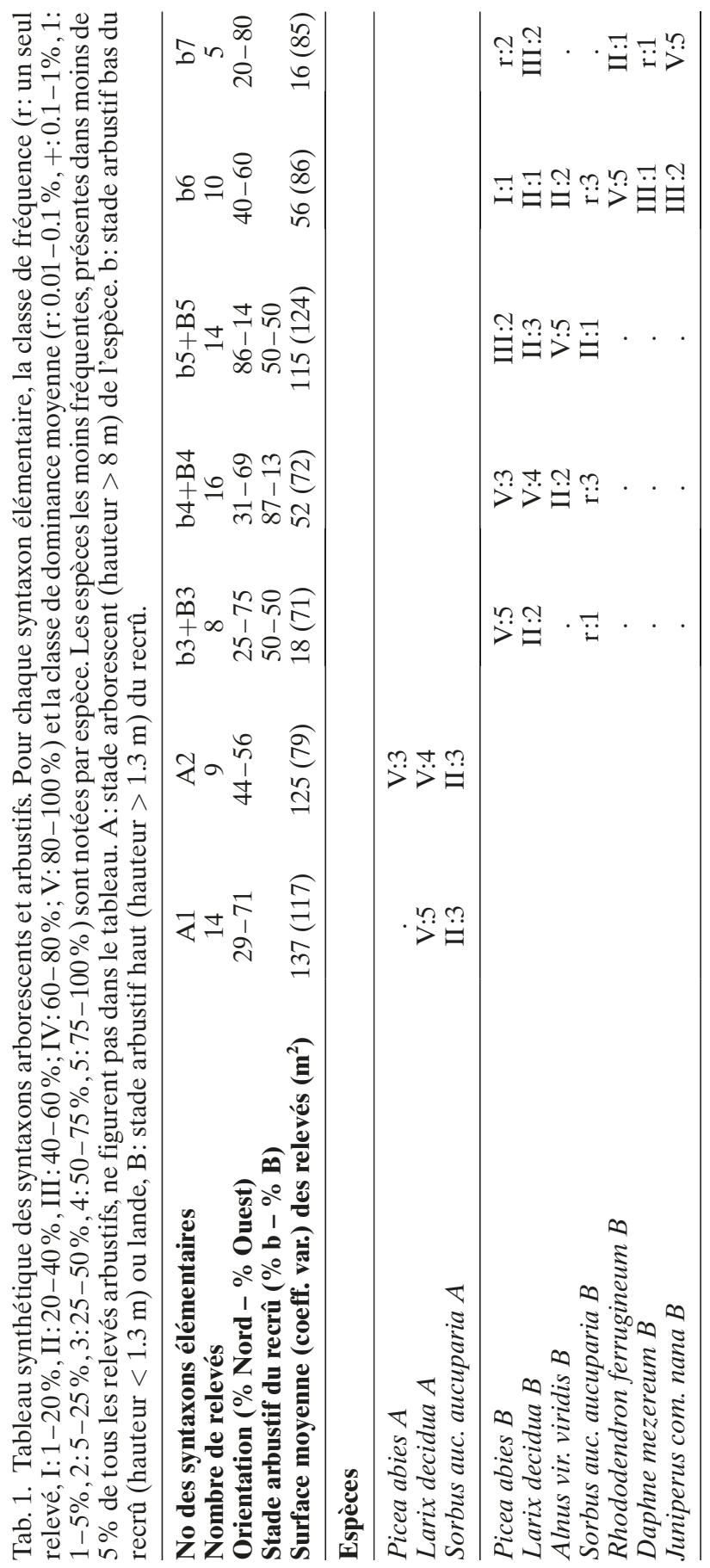


deux sous-arbrisseaux non abroutis par le bétail. En orientation nord et ouest, le premier domine (Tab. 1, syntaxon b6) et est accompagné du second et de Daphne mezereum, ainsi que du recrû d'Alnus viridis, de Larix et Picea. Le groupement à Juniperus communis ssp. nana (Tab. 1, syntaxon b7) prend le relais en orientation ouest principalement, en conditions plus xériques, accompagné de recrû de Larix.

\section{Typologie des synusies herbacées}

Sur les pentes en milieu ouvert, en orientation ouest et nord, se développe une pelouse acidocline et héliophile (Tab. 2, syntaxon h8) qui rassemble de nombreuses espèces à écologie bien contrastée. On y trouve, associées dans les mêmes relevés, des espèces calcifuges des pelouses et pâturages maigres d'altitude, caractéristiques des nardaies (Nardus stricta, Potentilla grandiflora, Campanula barbata, Pulsatilla alpina ssp. apiifolia, Arnica montana, Plantago alpina) avec des espèces calcicoles des pelouses sèches subalpines (Anthyllis vulneraria ssp. alpestris, Galium anisophyllon, Selaginella selaginoides, Lotus alpinus). Des plantes des prairies de fauche, des pâturages gras et des pelouses montagnardes complètent la composition floristique. Les espèces les plus fidèles sont (avec entre parenthèses l'indice IndVal et le degré de signification après un test par permutations aléatoires, $* * P<0.01$, *** $P<0.001)$ : Euphrasia rostkoviana (93.3***), Lotus alpinus (80.6***), Plantago alpina (80.0***), Nardus stricta (77.7***), Leontodon hispidus (75.1***), Plantago media (66.7***) et Leontodon pyrenaicus ssp. helveticus $\left(66.7^{* * *}\right)$. En situation de faible pente, sur les sentiers piétinés, quelques relevés dominés par un gazon dense de Nardus stricta ont été rattachés à ce groupe.

En orientation ouest exclusivement, à l'ombre d'une plus forte densité de Larix, un groupement de pelouse-ourlet acidophile (Tab. 2, syntaxon h9) proche du précédent, riche en espèces, montre une fréquence maximale de Pulsatilla alpina ssp. apiifolia et d'Arnica montana qui soulignent le fort apparentement avec les nardaies. Les espèces des lisières et ourlets secs et maigres et des sous-bois forestiers sont nombreuses mais peu recouvrantes. Les espèces des mégaphorbiaies et des marais ou prairies humides sont également bien présentes. Les espèces les plus fidèles sont Pulsatilla alpina ssp. apiifolia (83.2***), Phyteuma betonicifolium (61.9**), Larix au stade herbacé (58.2**), Centaurea montana (57.2**), Trifolium alpestre (54.4**) et Arnica montana (53.7**).

En orientation nord principalement, on trouve un groupement de sous-bois pâturé (Tab. 2, syntaxon h10) moins riche en espèces que les deux précédents, comprenant encore des espèces des pelouses, des pâturages et des prairies subalpines, accompagnées de nombreuses espèces des sous-bois forestiers, des mégaphorbiaies et des microphorbiaies. Les espèces les plus fidèles sont Viola biflora (81.0**), Homogyne alpina $\left(74.8^{* * *}\right)$ et Phyteuma spicatum $\left(57.6^{* *}\right)$.

Enfin, le dernier groupement herbacé est une mégaphorbiaie (Tab. 2, syntaxon H11) liée aux fourrés d'Alnus viridis, peu pâturés et orientés au nord. Ce syntaxon se distingue par sa physionomie de friche haute, par une richesse floristique moyenne plus faible, ainsi que par la fréquence et le recouvrement de Veratrum album, espèce hygrophile et nitrophile. Les hautes herbes des mégaphorbiaies sont bien présentes (Adenostyles alliariae, Peucedanum ostruthium) et les espèces des pelouses et pâturages sont rares. Les espèces les plus fidèles sont Veratrum album $\left(92.4^{* * *}\right)$ et Peucedanum ostruthium $(50.4 *)$. 


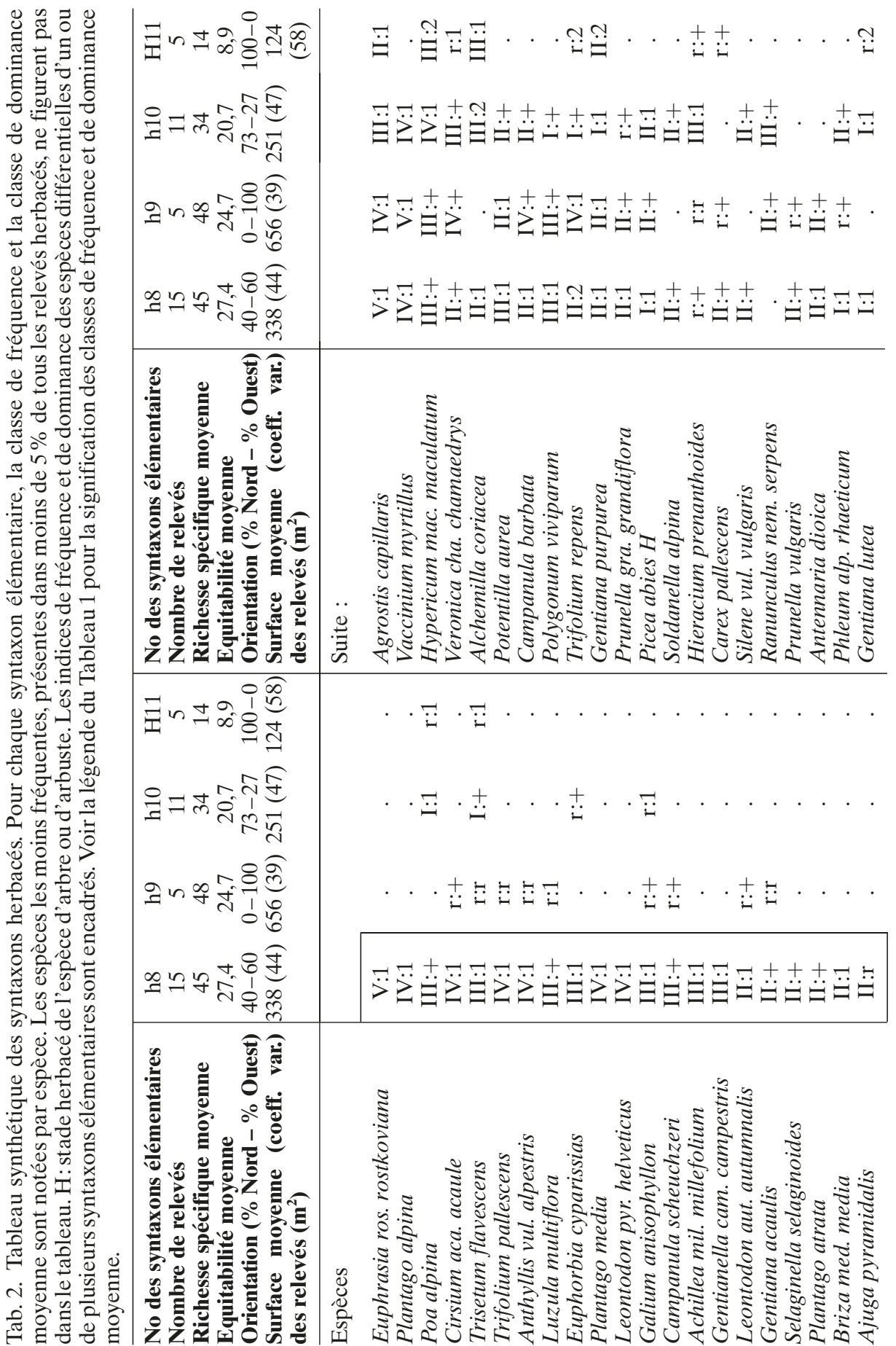




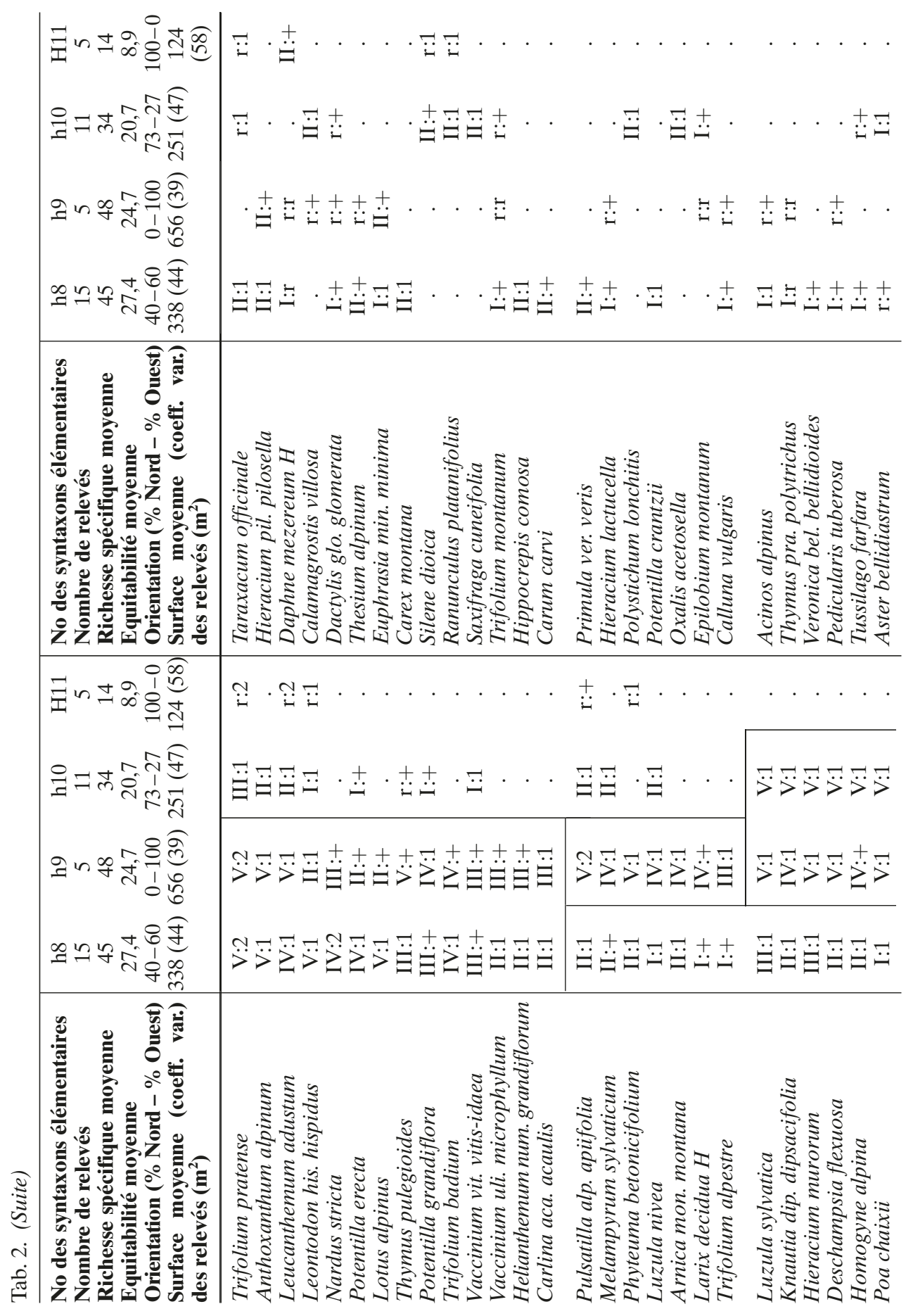




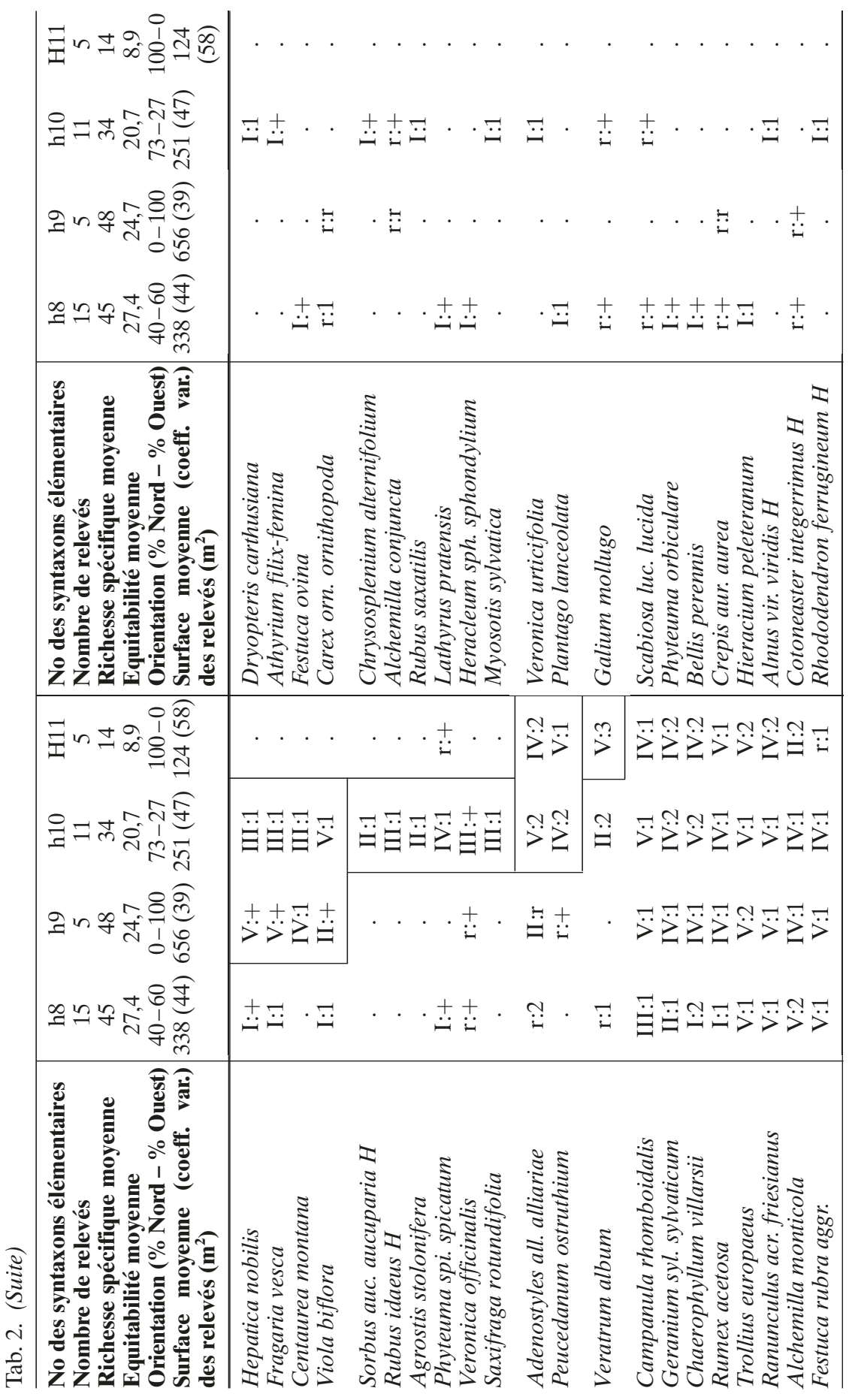




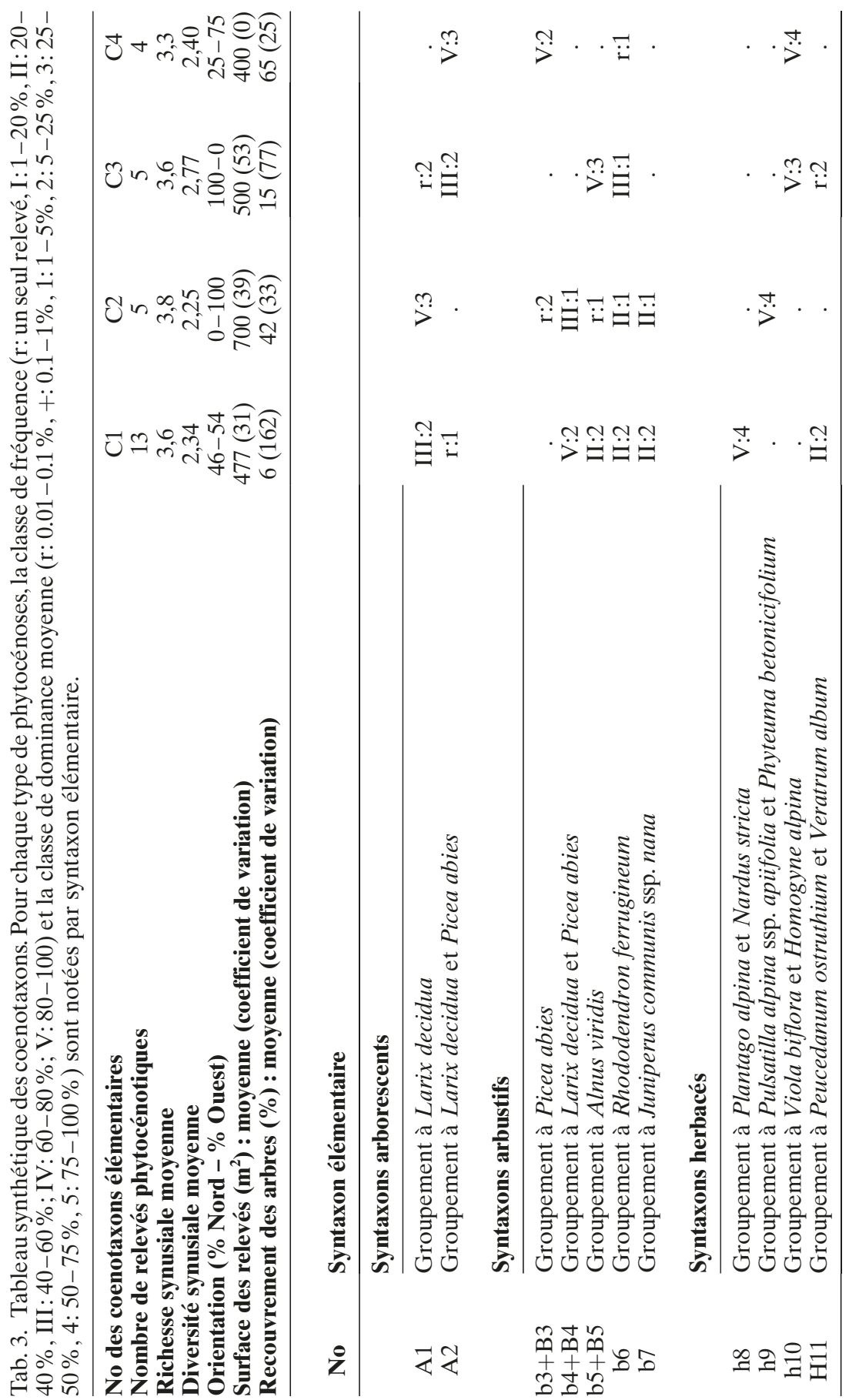




\section{Typologie des phytocénoses}

L'analyse des relevés phytocénotiques a fait apparaître quatre types bien caractérisés et aisément identifiables sur le terrain.

Le premier type (Tab. 3, coenotaxon C1) regroupe des phytocénoses de pâturages non ou peu boisés ( $6 \%$ en moyenne) avec Larix isolés (A1), en orientation ouest ou nord. Il est bien pâturé et présente toujours de nombreux Larix et Picea (b4+B4), les signes d'une initiation de la dynamique forestière. La pelouse à Nardus stricta enrichie de nombreuses espèces ( $\mathrm{h} 8$ ) est constante et dominante. Les espèces des pelouses maigres et des prés pâturés représentent près des trois quarts du recouvrement total de la strate herbacée (Tab. 4).

Tab. 4. Contribution des groupes socio-écologiques d'espèces herbacées dans les quatre types de phytocénoses, en pourcentage du recouvrement total.

\begin{tabular}{|c|c|c|c|c|}
\hline & \multicolumn{4}{|c|}{ Coenotaxon } \\
\hline & $\mathrm{C} 1$ & $\mathrm{C} 2$ & $\mathrm{C} 3$ & $\mathrm{C} 4$ \\
\hline \multicolumn{5}{|l|}{ Groupe d'espèces : } \\
\hline Prairies fauchées & $4.0 \%$ & $3.9 \%$ & $9.3 \%$ & $3.6 \%$ \\
\hline Prés pâturés & $31.4 \%$ & $16.7 \%$ & $10.5 \%$ & $12.0 \%$ \\
\hline Pelouses maigres & $43.1 \%$ & $31.4 \%$ & $10.9 \%$ & $8.7 \%$ \\
\hline Ourlets maigres & $2.0 \%$ & $5.2 \%$ & $4.1 \%$ & $6.3 \%$ \\
\hline Mégaphorbiaies & $9.2 \%$ & $21.1 \%$ & $48.3 \%$ & $56.6 \%$ \\
\hline Sous-bois et landes & $5.7 \%$ & $6.9 \%$ & $7.6 \%$ & $4.3 \%$ \\
\hline Marais & $4.6 \%$ & $14.7 \%$ & $9.2 \%$ & $8.6 \%$ \\
\hline Total & $100.0 \%$ & $100.0 \%$ & $100.0 \%$ & $100.0 \%$ \\
\hline
\end{tabular}

Le second type (Tab. 3, coenotaxon C2) regroupe des phytocénoses de pâturages très boisés à Larix, présents seulement en exposition ouest. En revanche, la synusie du recrû de Larix (b4+B4) est moins fréquente et moins dominante que dans le coenotaxon précédent. La strate herbacée est une pelouse-ourlet riche en espèces forestières (h9). Les espèces des pelouses maigres et des prés pâturés représentent environ la moitié du recouvrement total de la strate herbacée (Tab. 4).

Le troisième (Tab. 3, coenotaxon C3) correspond aux fourrés d'Alnus viridis (b5+B5), avec des îlots boisés de Larix et Picea (A2) que l'on trouve essentiellement en exposition nord. Le recrû de Picea est absent, mais cette espèce a été quelquefois relevée dans la strate herbacée. Celle-ci est essentiellement constituée de sous-bois (h10) et de mégaphorbiaies (H11), avec un recouvrement prédominant du groupe d'espèces des mégaphorbiaies et des microphorbiaies (Tab. 4).

Le dernier type (Tab.3, coenotaxon C4), constitué de relevés effectués le plus souvent hors des limites du pâturage actuel, en exposition nord et ouest, représente un stade de transition vers la forêt. Le taux de boisement est important, $65 \%$ en moyenne. Picea occupe une place prépondérante dans les strates arborescente et arbustive (b3+B3 et A2). Le sous-bois herbacé (h10) est dominé par les espèces des mégaphorbiaies, mais contient encore une bonne proportion d'espèces relictuelles des pâturages (Tab. 4). Il est à noter que la synusie d'Alnus viridis (b5+B5) n'a pas été relevée dans ces phytocénoses. 


\section{Biodiversité végétale}

Au niveau des communautés herbacées, la richesse floristique (Fig. 2) est généralement plus élevée dans les syntaxons de pelouse (h8) et de pelouse-ourlet (h9) que dans les sous-bois (h10) et les mégaphorbiaies (H11). Par contre, la régularité (Fig. 2) ne montre pas de différences appréciables entre les syntaxons, mais elle est très variable d'un relevé à l'autre pour un même type. Au niveau des phytocénoses, la diversité synusiale (Fig. 3) est très variable pour le coenotaxon $\mathrm{C} 1$ et elle est maximale pour le

Richesse floristique

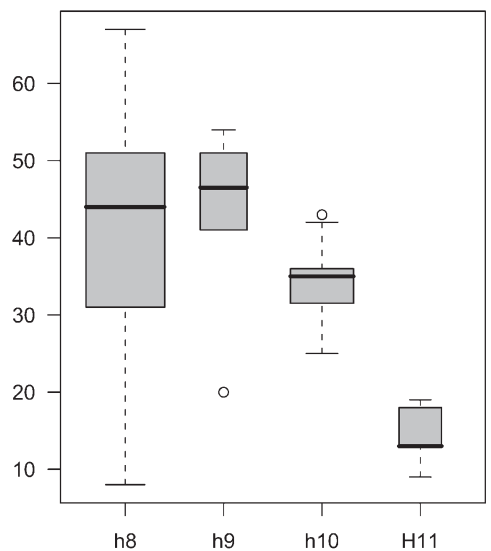

Régularité

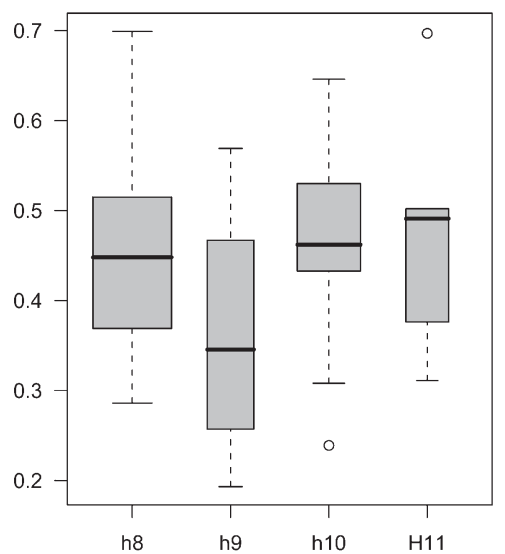

Fig. 2. Distribution des valeurs de la richesse floristique et la régularité des synusies herbacées selon leur type.
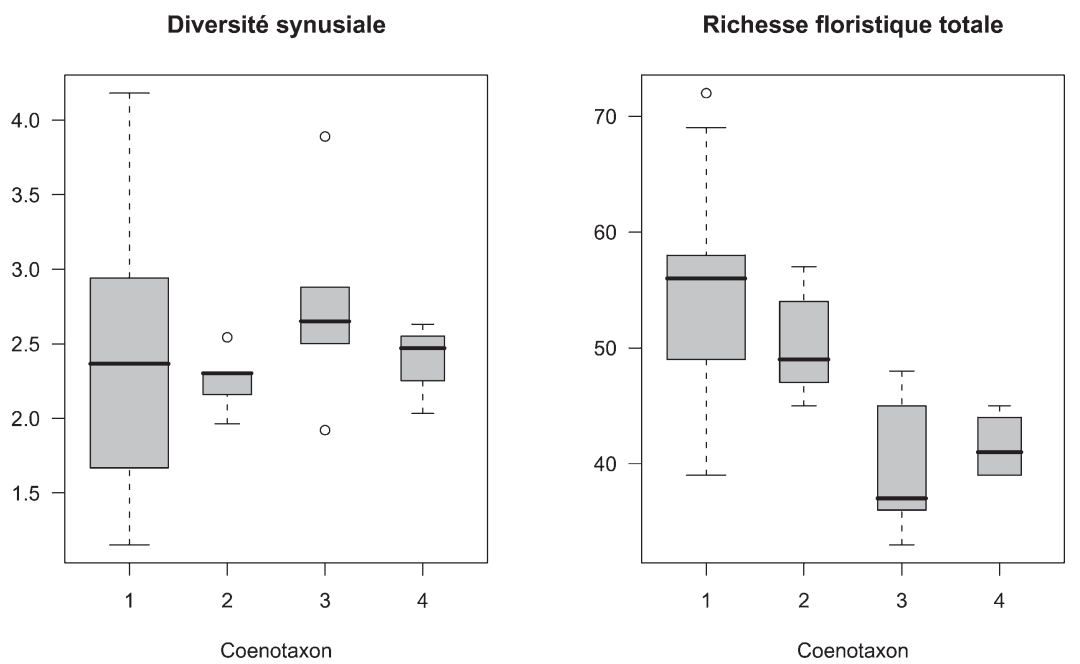

Fig. 3. Distribution des valeurs de la diversité synusiale et de la richesse floristique totale des phytocénoses selon leur type. 
coenotaxon C3, en raison de l'hétérogénéité structurelle des fourrés d'aulne vert. Par contre, la richesse floristique totale (Fig. 3) de plantes vasculaires est plus faible dans ce type de phytocénoses, et généralement moins élevé que dans le stade préforestier $(\mathrm{C} 4)$.

\section{Discussion}

\section{Syntaxons élémentaires}

La typologie des synusies arbustives (Tab. 1) nous a permis de séparer des groupements fonctionnellement différents. Trois syntaxons sont très importants car ils sont à l'origine de la dynamique du boisement: b3+B3 (Picea), b4+B4 (Larix et Picea) et b5+B5 (Alnus viridis) et c'est essentiellement sur leurs formes basses (b3, b4, b5) que le bétail aura une action importante: abroutissement, piétinement, écorçage. Les syntaxons de landes à Rhododendron ferrugineum (b6) et à Juniperus commmunis ssp. nana (b7) sont parfois associés à du recrû et pourraient peut-être le favoriser par facilitation indirecte car les deux espèces (respect. toxique et piquante) sont évitées par le bétail.

Les synusies herbacées (Tab. 2), apparentées aux pelouses alpines sur silice et aux mégaphorbiaies sont toutefois assez mal différenciées en raison de la présence d'un grand nombre d'espèces, souvent mélangées intimement sur le terrain, sans former des assemblages clairement délimités. Cette structure horizontale et verticale très floue, inhabituelle dans ce type de milieu, trahit vraisemblablement une situation d'instabilité dynamique, due à un changement récent dans le régime des perturbations ou à une transition successionnelle.

\section{Dynamique de la végétation}

La typologie des synusies et des phytocénoses ont permis d'émettre les hypothèses suivantes: En exposition nord (Fig. 4a), l'activité régulière du bétail permet le maintien d'une formation ouverte correspondant à des pâturages non ou peu boisés (C1) avec une pelouse composite à Nardus stricta (h8), dans laquelle le recrû de Larix (b4), et les fourrés d'Alnus viridis (b5) sont réprimés par le piétinement et l'abroutissement. Les espèces de la mégaphorbiaies (H11) sont aussi limitées par l'action du bétail, sauf dans les endroits très riches en azotes où certaines espèces constituent une végétation de reposoirs. Les plus grands Larix sont généralement présents (B4, A1), mais isolés et peu recouvrants. La dynamique naturelle en cas d'abandon se marque par le développement rapide des fourrés d'Alnus viridis (b5, B5) provoquant une transition vers l'aulnaie verte (C3). Les conditions d'ombrage et d'humidité, accrue au niveau du sol, combinées à l'effet améliorant de la litière de l'aulne provoquent des transformations importantes dans la strate herbacée, favorisant les espèces des pâturages gras d'altitude et des mégaphorbiaies. Ces mêmes conditions freinent l'installation et le développement des mélèzes au profit des épicéas, beaucoup plus tolérants à l'ombrage et à l'hygrométrie. La succession progressive secondaire se termine par une formation préforestière (C4) dominée par Picea, accompagné de Larix (A2). Dans la strate arbustive, Larix et Alnus viridis, ne supportant pas l'ombrage, finissent par disparaître. La végétation herbacée diminue en nombre d'espèces, mais se modifie peu.

En exposition ouest (Fig. 4b), le stade initial et le stade final sont peu différents de la série dynamique précédente, alors que le stade intermédiaire est très différent, formé 
a) Versant exposé au nord

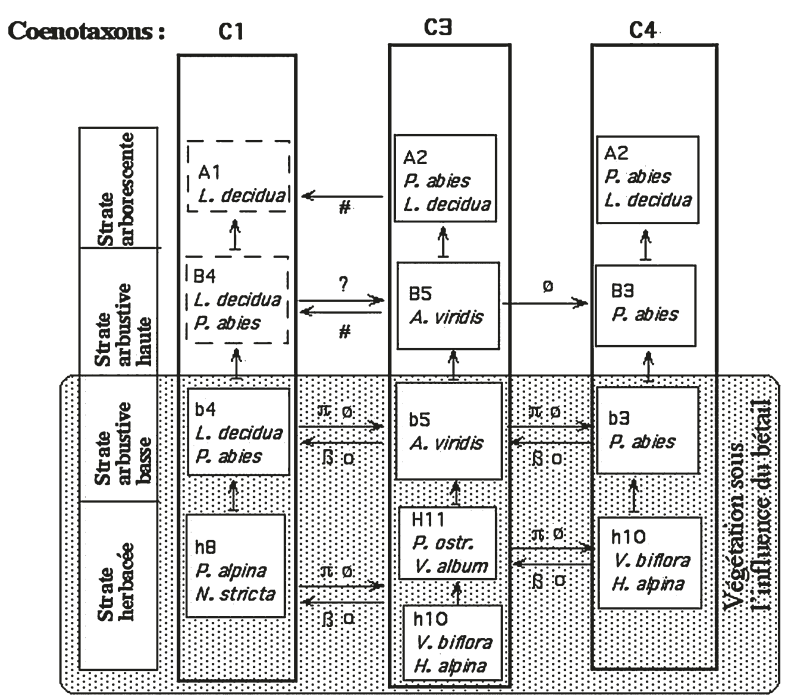

\section{b) Versant exposé à l'ouest}

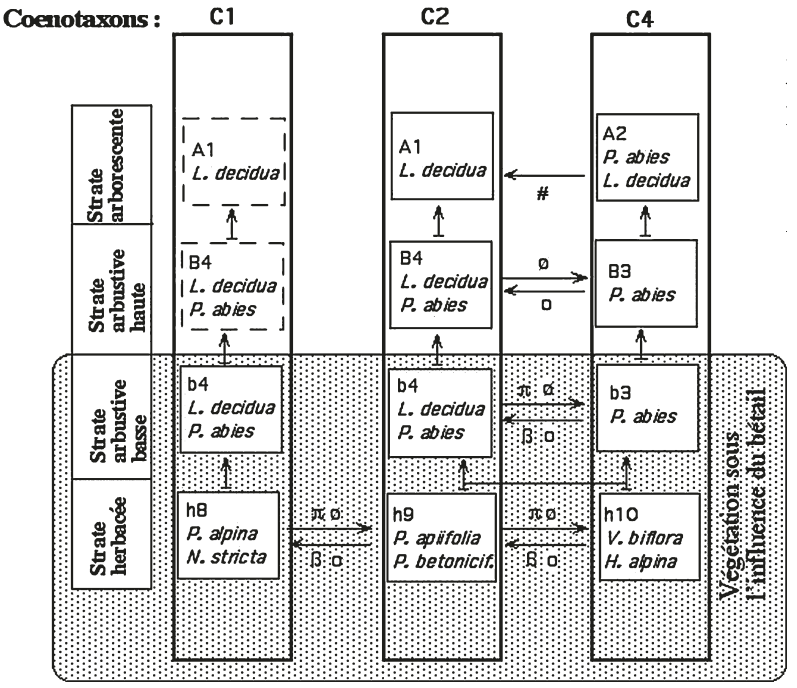

Fig. 4. Modèles qualitatifs hypothétiques de la dynamique de la végétation sur les versants nord (a) et ouest (b) de l'alpage du Larzay. Les flèches horizontales représentent les contraintes écologiques menant au remplacement d'une synusie dans une même strate. Les principales contraintes identifiées sont: $\varnothing$ : augmentation de l'ombrage, $\mathrm{o}$ : augmentation de la lumière, $B$ : augmentation de la pression de broutage, $\pi$ : diminution de la pression de broutage, \#: essartage des arbres du sous-système non pâturé. Les flèches verticales représentent le développement de la stratification dans les phytocénoses. Deux sous-ensembles sont décrits, l'un soumis au broutage (hauteur de la végétation $<1.3 \mathrm{~m}$ ), l'autre pas (hauteur $>1.3 \mathrm{~m}$ ). $\mathrm{b}$ : stade arbustif bas du recrû, B: stade arbustif haut du recrû. Cadres en traits tillés: syntaxons peu fréquents et peu recouvrants. 
d'un mélézein relativement dense, hébergeant une végétation herbacée de pelouseourlet enrichie en espèces forestières (h9). Le stade initial (C1) a montré localement une très forte dynamique d'installation du mélèze (b4).

\section{Diversité végétale}

Au cours de la dynamique de la végétation (de h8 à h10), la diversité des plantes vasculaires herbacées diminue de façon importante (env. $25 \%$ ) alors que la régularité varie très peu (Fig. 2). On notera un appauvrissement majeur en sous-bois de l'aulnaie verte (H11), lorsque celle-ci devient dense et que bétail n'y pâture plus. La plus grande variabilité de la régularité de $\mathrm{h} 8$ est liée à un faciès de la nardaie très appauvri en espèces où Nardus stricta peut devenir très couvrant (Fig. 2). Au niveau des phytocénoses, la richesse floristique totale (Fig. 3) diminue aussi de façon importante (env. $25 \%$ ) ou cours de la dynamique (de $\mathrm{Cl}$ à $\mathrm{C} 4$ ). Une valeur très faible pour l'aulnaie verte (C3) est à mettre en relation avec la dominance de H11 pauvres en espèces. En revanche, si la diversité synusiale (Fig. 3) varie peu au cours de l'évolution, on remarque une très grande variabilité pour les phytocénoses du type $\mathrm{C} 1$, signe d'une grande hétérogénéité structurelle, suivant la présence ou non des diverses synusies arbustives (landes, recrû bas et recrû haut) et des grands mélèzes (A1). La baisse de diversité en espèces est à mettre en relation avec l'augmentation du recouvrement des arbres qui passe de $6 \%(\mathrm{C} 1)$ à $65 \%(\mathrm{C} 4)$ en moyenne (Tab. 3$)$.

La baisse de diversité suite à l'abandon de prés de fauche ou de pâturages de montagne est rapportée pour les plantes vasculaires (Gillet et al. 1999; Pykälä 2003; Dullinger et al. 2003; Pavlu et al. 2005; Baur et al. 2006; Maurer et al. 2006) comme pour d'autres groupes d'organismes comme les lépidoptères (Balmer et Erhardt 2000), les hyménoptères-orthoptères-coléoptères (Anthelme et al. 2001), les oiseaux (Laiolo et al. 2004). La diversité structurelle de la végétation est également importante: la présence d'une mosaïque de synusies (herbacées, arbustives, arborescentes) de différentes hauteurs, abritant des conditions abiotiques spécifiques augmentent la probabilité d'apparition de nouvelles espèces ou communautés. L'utilisation des grands herbivores permet généralement d'entretenir une dynamique de la mosaïque et de maintenir ou rétablir la diversité de la végétation (Olff et Ritchie 1998; Olff et al. 1999; Dullinger et al. 2003; Pykälä 2003; Bakker et al. 2004). Il est cependant important d'éviter que l'utilisation ne se concentre sur une petite partie de l'alpage (Jewell et al. 2005). Un système de pâture tournante est proposé par Baur et al. (2006) pour maintenir une mosaïque de stades successionnels et pour assurer une bonne biodiversité.

\section{Conclusions et perspectives}

La méthode synusiale, réalisée par une approche synchronique sur les différents stades de boisement du site du Larzay, a permis d'évaluer non seulement la composition floristique, mais aussi la composante structurelle. Elle nous a aussi permis d'établir des schémas dynamiques et d'évaluer la perte de diversité de plantes entre les stades.

Bien que notre travail soit une étude de cas d'une surface très limitée en domaine siliceux, la problématique de l'embroussaillement par Alnus viridis, Larix et Picea et l'évolution vers la forêt fermée au Larzay peut certainement être généralisée à de nombreuses situations des Alpes, notamment dans les Alpes du nord (avec Alnus 
viridis) et les Alpes centrales (avec Larix). L'embuissonnement par ces deux espèces suite à une déprise, rapportés par d'autres auteurs (Anthelme et al. 2001; Anthelme et al. 2002; Didier 2001; Wiedmer et Senn-Irlet 2006), sont fréquents dans les Alpes et s'accompagnent, ici comme ailleurs, d'une perte de biodiversité. Les exemples de successions secondaires bien documentés (voir aussi Risch et al. 2004), avec les principales essences intervenant dans les dynamiques de boisement à l'étage subalpin méritent de nouvelles investigations.

En complément aux descriptions très fines de la végétation, il y a lieu de bien mettre en évidence les différentes actions du bétail sur les plantes et leurs communautés, en particulier sur les jeunes arbres et leurs synusies arborescentes basses (Vandenberghe et al. 2007) qui constituent le point de départ de la dynamique du boisement. De bonnes mesures de gestion, des solutions innovantes de conduite des troupeaux avec un bétail adapté permettront d'assurer à long terme un paysage varié et diversifié, issu d'un pastoralisme séculaire.

\section{Résumé}

La réduction de pression de pâture dans les Alpes est à l'origine de l'embuissonnement de nombreuses pâtures depuis quelques décennies. Pour comprendre la dynamique de colonisation naturelle de la forêt et pour évaluer comment ces changements affectent la diversité en espèces de plantes, nous avons décrit la végétation d'un pâturage boisé des Alpes centrales suisses (Sembrancher, Valais) en utilisant la méthode synusiale intégrée. Sur la base de 27 placettes choisies de façon stratifiée, nous avons défini 11 types de communautés au niveau synusial (deux types de synusies arborescentes, cinq d'arbustives, et quatre d'herbacées) et quatre types de communautés au niveau des phytocénoses (pâturage, aulnaie verte et mégaphorbiaie, pâturage boisé et jeune forêt). La distribution spatiale de ces quatre phytocénoses suggère qu'elle représentent des stades successionnels après l'abandon et que le patron de succession dépend de l'exposition. Nous supposons qu'en exposition nord, en situations fraîches et ombragées, les pâtures abandonnées se développent d'abord vers une prairie à grandes herbes et buissons d'Alnus viridis et ensuite vers un stade préforestier à Picea abies et Larix decidua. En orientation ouest, en situations chaudes et ensoleillées, Larix decidua (principalement) et Picea abies colonisent le pâturage abandonné et la succession secondaire converge finalement vers le même stade préforestier qu'en exposition nord. La richesse en plantes vasculaires était maximale dans les zones ouvertes et diminuait de $25 \%$ alors que le taux de boisement passait de $6 \%$ à $65 \%$. D'après notre modèle de succession, la diversité en espèces de plantes est perdue plus rapidement en exposition nord (avec des fourrés d'aulne vert pauvres en espèces) qu'en exposition ouest (avec de jeunes forêts de mélèze riches en espèces), suggérant que les pentes exposées au nord ont le plus urgemment besoin d'une gestion appropriée de la pâture.

Nous remercions les institutions suivantes pour leur soutien financier au projet PASTO: la fondation MAVA, l'Etat du Valais, l'Office fédéral de l'environnement (OFEV), la Loterie romande et la Fédération d'élevage de la race d'Hérens. Nous adressons notre gratitude à Martine Rebetez et feu Martin Burkhard pour divers renseignements fournis sur le climat et la géologie, ainsi qu'à Mélanie Viret et Bernard Jeangros pour leur lecture d'une première version du manuscrit. Les commentaires et suggestions de Sabine Güsewell et des deux reviewers ont permis d'améliorer sensiblement le manuscrit; nous les en remercions. Nous remercions aussi Charlotte Vandenberghe pour sa relecture du résumé en anglais. 


\section{Références}

Anthelme F., Grossi J.L., Brun J.J. and Didier L. 2001. Consequences of green alder expansion on vegetation changes and arthropod communities removal in the northern French Alps. For. Ecol. Manage. 145: 57 - 65.

Anthelme F., Cornillon L. and Brun J.J. 2002. Secondary succession of Alnus viridis (Chaix) DC. in Vanoise National Park, France: coexistence of sexual and vegetative strategies. Ann. For. Sci. 59: $419-428$.

Bakker E.S., Olff H., Vandenberghe C., de Maeyer K., Smit R., Gleichman J.M. and Vera F.W.M. 2004. Ecological anachronisms in the recruitment of temperate light-demanding tree species in wooded pastures. J. Appl. Ecol. 41: 571 - 582.

Balmer O. and Erhardt A. 2000. Consequences of succession on extensively grazed grasslands for central European butterfly communities: Rethinking conservation practices. Conserv. Biol. 14: 746 - 757.

Baur B., Cremene C., Groza G., Rakosy L., Schileyko A.A., Baur A., Stoll P. and Erhardt A. 2006. Effects of abandonment of subalpine hay meadows on plant and invertebrate diversity in Transylvania, Romania. Biol. Conserv. 132: 261 - 273.

Braun-Blanquet J. 1964. Pflanzensoziologie. Grundzüge der Vegetationskunde. Springer, Wien.

Burri M., Jemelin N., Oulianoff N., Ayrton S., Blanc P., Grasmück K., Krummenacher, von Raumer J.-F., Stalder P., Trümpy R. et Wutzler B. 1983. Atlas Géologique Suisse 1:25’000, ISSN 1420 - 2905, No. 77: Carte Nationale 1325, Sembrancher. Office Fédéral des eaux et de la Géologie.

Delarze R., Gonseth Y. et Galland P. 1998. Guide des milieux naturels de Suisse. Delachaux et Niestlé, Lausanne.

Didier L. 2001. Invasion patterns of European larch and Swiss stone pine in subalpine pastures in the French Alps. For. Ecol. and Manage. 145: 67 - 77.

Dufrêne M. and Legendre P. 1997. Species assemblages and indicator species: The need for a flexible asymmetrical approach. Ecol. Monogr. 67: 345 - 366.

Dullinger S., Dirnbock T., Greimler J. and Grabherr G. 2003. A resampling approach for evaluating effects of pasture abandonment on subalpine plant species diversity. J. Veg. Sci. 14: $243-252$.

Favarger C. 1995. Flore et végétation des Alpes. Tome 2. Delachaux et Niestlé, Lausanne.

Fellay E. 1998. La race d'Hérens. Revue suisse Agric. 30: 2 - 7.

Fischer M. and Wipf S. 2002. Effect of low-intensity grazing on the species-rich vegetation of traditionally mown subalpine meadows. Biol. Conserv. 104: 1 - 11 .

Gillet F., de Foucault B. et Julve P. 1991. La phytosociologie synusiale intégrée: objets et concepts. Candollea 46: $315-340$.

Gillet F. and Gallandat J.D. 1996. Integrated synusial phytosociology: some notes on a new multiscalar approach to vegetation analysis. J. Veg. Sci. 7: $13-18$.

Gillet F., Murisier B., Buttler A., Gallandat J.D. and Gobat J.M. 1999. Influence of tree cover on the diversity of herbaceous communities in subalpine wooded pastures. Appl. Veg. Sci. 2: $47-54$.

Jewell P.L., Güsewell S., Berry N.R., Käuferle D., Kreuzer M. and Edwards P.J. 2005. Vegetation patterns maintained by cattle grazing on a degraded mountain pasture. Bot. Helv. 115: 109 - 124.

Julve P. 1993. Synopsis phytosociologique de la France (communautés de plantes vasculaires). Lejeunia 140: 1 - 160 .

Julve P. 2006. Baseflor: Index botanique, écologique et chorologique de la flore de France. http://perso.orange.fr/philippe.julve/catminat.htm

Laiolo P., Dondero F., Ciliento E. and Rolando A. 2004. Consequences of pastoral abandonment for the structure and diversity of the alpine avifauna. J. Appl. Ecol. 41: 294 - 304

Legendre P. and Legendre L. 1998. Numerical Ecology, $2^{\text {nd }}$ ed. Elsevier, Amsterdam. 
Maurer K., Weyand A., Fischer M. and Stöcklin J. 2006. Old cultural traditions, in addition to land use and topography, are shaping plant diversity of grasslands in the Alps. Biol. Conserv. 130: $438-446$.

Oberdorfer E. 1992. Süddeutsche Pflanzengesellschaften, Teil IV. Fischer, Jena.

OFS/OFEFP 2004. La forêt et le bois. Annuaire 2004. Surface forestière.

OFS 2005. Communiqué de presse No 0351 - 0511 - 50 du 25 novembre 2005.

Olff H. and Ritchie M.E. 1998. Effects of herbivores on grassland plant diversity. Trends Ecol. Evol. 13: $261-265$.

Olff H., Vera F.W.M., Bokdam J., Bakker E.S., Gleichman J.M., de Maeyer K. and Smit R. 1999. Shifting mosaics in grazed woodlands driven by the alternation of plant facilitation and competition. Plant Biol. 1: 127 - 137.

Ozenda P. 1985. La végétation de la chaîne alpine. Masson, Paris.

Pavlu V., Hejcman M., Pavlu L., Gaisler J., Nezerkova P. and Andaluz M.G. 2005. Vegetation changes after cessation of grazing management in the Jizerske Mountains (Czech Republic). Ann. Bot. Fenn. 42:343 - 349.

Pykälä J. 2003. Effects of restoration with cattle grazing on plant species composition and richness of semi-natural grasslands. Biodivers. Conserv. 12: $2211-2226$.

R Development Core Team 2006. R: A language and environment for statistical computing. R Foundation for Statistical Computing, Vienna, Austria. URL http://www.R-project.org.

Risch A.C., Schutz M., Krüsi B.O., Kienast F., Wildi O. and Bugmann H. 2004. Detecting successional changes in long-term empirical data from subalpine conifer forests. Plant Ecol. 172: 95 - 105.

Richard L. 1990. Ecologie des mégaphorbiaies subalpines à aune vert de la Vannoise et des régions environnantes. Trav. Sci. Parc Nat. Vannoise 17: 127 - 158.

Rousseeuw P.J. 1987. Silhouettes: A graphical aid to the interpretation and validation of cluster analysis. J. Comput. Appl. Math. 20: 53 - 65.

Tasser E. and Tappeiner U. 2002. Impact of land use changes on mountain vegetation. Appl. Veg. Sci. 5: $173-184$

Tukey J.W. 1977. Exploratory Data Analysis. Addison-Wesley, Reading, MA.

Tutin T.G., Heywood V.H., Burges N.A., Valentine D.H., Walters S.M. and Webb D.A. (eds.) 1964 - 1980. Flora Europaea, 1 - 5. Cambridge University Press, Cambridge.

Vandenberghe C., Freléchoux F., Moravie M.-A., Gadallah F. and Buttler A. 2007. Short-term effects of cattle browsing on tree sapling growth in mountain wooded pastures. Plant Ecol. 188: $253-264$.

Werner P. 1988. La Flore, collection Connaître la nature en Valais. Editions Pillet, Martigny.

Wiedmer E. and Senn-Irlet B. 2006. Biomass and primary productivity of an Alnus viridis stand - a case study from the Schachental valley, Switzerland. Bot. Helv. 116: 55 - 64. 